\title{
INTEGRAL PRICE FORMULAS FOR LOOKBACK OPTIONS
}

\author{
CHENGLONG XU AND YUE KUEN KWOK
}

Received 6 August 2004 and in revised form 5 November 2004

We derive an integral representation of the price formulas for European options whose terminal payoff involves path-dependent lookback variable. The intricacies in the derivation procedures using the partial differential equation techniques stem from the degenerate nature of the pricing models, where the lookback state variables appear only in the auxiliary conditions but not in the governing differential equations. We also derive a parity relation between the price functions of the floating strike and fixed strike lookback options.

\section{Background and model formulation}

The mathematical formulation for the price function of an option whose terminal payoff involves path dependent lookback variable has been quite well explored in the literature. Let $S$ denote the stock price variable and $M$ denote the maximum price variable. Here, $M$ represents the realized maximum of the stock price recorded from the initial time of the lookback period to the current time. Let $t$ denote the calendar time variable, $T$ the maturity date of the lookback option, and $\tau=T-t$ the time to expiry. Under the Black-Scholes framework, the partial differential equation formulation for the price function $V(S, M, \tau)$ of the one-asset European lookback option model with terminal payoff $V_{T}(S, M)$ is given by (Goldman et al. [3])

$$
\begin{gathered}
\frac{\partial V}{\partial \tau}=\frac{\sigma^{2}}{2} S^{2} \frac{\partial^{2} V}{\partial S^{2}}+r S \frac{\partial V}{\partial S}-r V, \quad 0<S<M, \tau>0 \\
\left.\frac{\partial V}{\partial M}\right|_{S=M}=0, \quad \tau>0 \\
V(S, M, 0)=V_{T}(S, M)
\end{gathered}
$$

where $r$ is the riskless interest rate and $\sigma$ is the volatility of the stock price. For the sake of simplicity, we assume the stock to be zero dividend paying. The price function is essentially two-dimensional with state variables $S$ and $M$. However, the differential equation exhibits the degenerate nature in the sense that it does not involve the lookback 
variable $M$. Here, $M$ only occurs in the Neumann boundary condition $\partial V /\left.\partial M\right|_{S=M}=0$ and the terminal payoff function. The Neumann boundary condition signifies that if the current stock price equals the value of the current realized maximum, then the option price is insensitive to $M$. The proof of uniqueness of a solution to the lookback option model cannot be inferred directly from similar uniqueness properties of parabolic differential equations due to the degenerate nature of the governing differential equation. The uniqueness issue in the sense of viscosity solution of the lookback option model has been addressed by Barles [1].

Lookback option prices are commonly evaluated using the probability approach, where the option value is obtained as the discounted expectation of the terminal payoff under the risk neutral measure. The solution procedure requires the determination of the density function of the joint processes of the stock price and its realized maximum (Conze and Viswanathan [2]; He et al. [4]). For lookback options with simple payoff function, like the common fixed strike and floating strike lookback options, the expectation calculations can be performed in a straightforward manner. When one deals with a general payoff $V_{T}(S, M)$, the integral representation of the price function derived using the probability approach would become much cumbersome. However, our calculations show that the integral representation obtained using the partial differential equation approach appears to be quite succinct and concise.

In this paper, we demonstrate the use of the partial differential equation techniques to derive general integral price formulas for lookback option models. First, we reformulate the pricing model (1.1) using the following new set of variables:

$$
x=\ln \frac{M}{S}, \quad y=\ln M
$$

With the new set of variables, the lookback pricing model formulation can be rewritten as

$$
\begin{gathered}
\frac{\partial V}{\partial \tau}=\frac{\sigma^{2}}{2} \frac{\partial^{2} V}{\partial x^{2}}-\left(r-\frac{\sigma^{2}}{2}\right) \frac{\partial V}{\partial x}, \quad x>0,-\infty<y<\infty, \tau>0 \\
\left.\left(\frac{\partial V}{\partial x}+\frac{\partial V}{\partial y}\right)\right|_{x=0}=0, \quad \tau>0 \\
V(x, y, 0)=V_{T}\left(e^{y-x}, e^{y}\right) .
\end{gathered}
$$

The triangular wedge shape of the original domain of definition $\mathscr{D}=\{(S, M): 0<S<M\}$ is now transformed into a new domain which is the semi-infinite two-dimensional plane $\tilde{\mathscr{D}}=\{(x, y): x>0$ and $-\infty<y<\infty\}$. However, the boundary condition along $x=0$ involves the function $\partial V / \partial x+\partial V / \partial y$.

In the next section, we derive the integral price formulas for one-asset European options with general lookback payoff functions. In Section 3, we deduce a parity relation between the price functions of the floating strike and fixed strike lookback options. The paper ends with conclusive remarks in Section 4. 


\section{Integral price formulas for European lookback options}

In this section, we derive the integral price formula for the pricing model (1.3). The difficulties in the derivation procedure arise from the boundary condition along $x=0$, which involves $\partial V / \partial x+\partial V / \partial y$.

We define the function

$$
W(x, y, \tau)=\frac{\partial V}{\partial x}+\frac{\partial V}{\partial y},
$$

and in terms of $W(x, y, \tau),(1.3)$ can be rewritten as

$$
\begin{gathered}
\frac{\partial W}{\partial \tau}=\frac{\sigma^{2}}{2} \frac{\partial^{2} W}{\partial x^{2}}-\left(r+\frac{\sigma^{2}}{2}\right) \frac{\partial W}{\partial x}, \quad x>0,-\infty<y<\infty, \tau>0, \\
W(0, y, \tau)=0, \quad \tau>0, \\
W(x, y, 0)=\left(\frac{\partial}{\partial x}+\frac{\partial}{\partial y}\right) V_{T}\left(e^{y-x}, e^{y}\right) .
\end{gathered}
$$

The variable $y$ appears only as a parameter in the above formulation. Hence, the solution of $W(x, y, \tau)$ is seen to be

$$
W(x, y, \tau)=\int_{0}^{\infty} \bar{G}(\xi, \tau ; x) W(\xi, y, 0) d \xi
$$

where the Green function $\bar{G}(\xi, \tau ; x)$ corresponding to the semi-infinite domain $\tilde{\mathscr{D}}$ is given by

$$
\bar{G}(\xi, \tau ; x)=[\psi(x-\xi, \tau)-\psi(x+\xi, \tau)] e^{\alpha(x-\xi)+\beta \tau}
$$

with

$$
\alpha=\frac{r}{\sigma^{2}}-\frac{1}{2}, \quad \beta=-\frac{1}{2 \sigma^{2}}\left(r-\frac{\sigma^{2}}{2}\right)^{2}, \quad \psi(x, \tau)=\frac{1}{\sigma \sqrt{2 \pi \tau}} \exp \left(-\frac{x^{2}}{2 \sigma^{2} \tau}\right) .
$$

Once $W(x, y, \tau)$ is known, we then solve for $V(x, y, \tau)$ using (2.1). First, we may rewrite (2.1) as

$$
W(\xi, \xi+y-x, \tau)=\frac{d}{d \xi} V(\xi, \xi+y-x, \tau), \quad \forall \xi>0 .
$$

Upon integrating with respect to $\xi$ from 0 to $x$, we obtain

$$
V(x, y, \tau)=V(0, y-x, \tau)+\int_{0}^{x} W(\xi, \xi+y-x, \tau) d \xi
$$


The remaining step amounts to the determination of $V(0, y-x, \tau)$. Suppose we write $\phi(z, \tau)=V(0,-z, \tau)$, where $z=x-y$, it can be shown that $\phi$ satisfies

$$
\begin{gathered}
\frac{\partial \phi}{\partial \tau}=\frac{\sigma^{2}}{2} \frac{\partial^{2} \phi}{\partial z^{2}}-\left(r-\frac{\sigma^{2}}{2}\right) \frac{\partial \phi}{\partial z}+\frac{\sigma^{2}}{2} \frac{\partial W}{\partial x}(0,-z, \tau), \quad-\infty<z<\infty, \tau>0 \\
\phi(z, 0)=U(0,-z, 0)=V_{T}\left(e^{-z}, e^{-z}\right) .
\end{gathered}
$$

If we use $G(\eta, \tau ; z)$ to denote the infinite domain Green function of the above problem,

$$
G(\eta, \tau ; z)=e^{\alpha(z-\eta)+\beta \tau} \psi(z-\eta, \tau)
$$

then the solution to $\phi(z, \tau)$ can be formally represented by

$$
\begin{aligned}
\phi(z, \tau)= & \int_{0}^{\tau} \int_{-\infty}^{\infty} G(-\eta, \tau-u ; z) \frac{\sigma^{2}}{2} \frac{\partial W}{\partial x}(0, \eta, u) d \eta d u \\
& +\int_{-\infty}^{\infty} G(-\eta, \tau ; z) V_{T}\left(e^{\eta}, e^{\eta}\right) d \eta .
\end{aligned}
$$

The integrand in the double integral still involves $\partial W / \partial x$. It would be more desirable to transform the double integral into the form that involves $V_{T}$ only. By performing some analytic calculations (see Appendix A for the details), we obtain

$$
\begin{aligned}
V(S, M, \tau)= & \int_{0}^{\infty} \bar{G}\left(\xi, \tau ; \ln \frac{M}{S}\right) V_{T}\left(M e^{-\xi}, M\right) d \xi \\
& +\int_{0}^{\infty} \int_{\ln M}^{\infty}\left[\left(\frac{\partial}{\partial \xi}+\frac{\partial}{\partial \eta}\right) \bar{G}\left(\xi, \tau ; \eta+\ln \frac{1}{S}\right)\right] V_{T}\left(e^{\eta-\xi}, e^{\eta}\right) d \eta d \xi
\end{aligned}
$$

It is relatively straightforward to show that the above solution satisfies the differential equation and auxiliary conditions as stated in (1.3).

\section{Floating strike and fixed strike lookback options}

The integral price formula (2.10) gives the value of a lookback option with general terminal payoff function $V_{T}(S, M)$. The two most common lookback options have payoff of the form: (i) floating strike payoff, $M-S$; (ii) fixed strike payoff, $\max (M-K, 0)$, where $K$ is the fixed strike price. In this section, we first consider the valuation of lookback options with payoff of the form $S f(M / S)$, which includes the floating strike payoff as a special example. We illustrate how to achieve dimension reduction of the pricing model under this special form of terminal payoff. Then, we deduce the parity relation between the option values of floating strike and fixed strike lookback options.

By taking $V_{T}(S, M)=S f(M / S)$ and applying the transformations of the variables $x=$ $\ln (M / S)$ and $U(x, \tau)=V(S, M, \tau) / S$ to the pricing formulation (1.1), we obtain

$$
\begin{gathered}
\frac{\partial U}{\partial \tau}=\frac{\sigma^{2}}{2} \frac{\partial^{2} U}{\partial x^{2}}-\left(r+\frac{\sigma^{2}}{2}\right) \frac{\partial U}{\partial x}, \quad x>0, \tau>0, \\
\left.\frac{\partial U}{\partial x}\right|_{x=0}=0, \quad \tau>0 \\
U(x, 0)=f\left(e^{x}\right) .
\end{gathered}
$$


The new formulation involves only one-space variable, so dimension reduction has been achieved. To resolve the difficulty of dealing with the Neumann boundary condition along $x=0$, we extend the domain of definition from the semi-infinite domain to the fullinfinite domain. This is achieved by performing continuation of the initial condition to the domain $x<0$ such that the price function can satisfy the Neumann boundary condition. Due to the presence of the drift term in the differential equation, the simple oddeven extension is not applicable. In Appendix B, we present the details of the construction of the continuation function. For example, for the floating strike payoff $M-S$, we have $U(x, 0)=e^{x}-1, x>0$. The continuation of the initial condition to the domain $x<0$ is found to be (see Appendix B)

$$
U(x, 0)=\frac{1-e^{(2 \tilde{\alpha}-1) x}}{2 \tilde{\alpha}-1}, \quad x<0, \tilde{\alpha}=\frac{r}{\sigma^{2}}+\frac{1}{2} .
$$

We obtain the integral price formula of lookback option with payoff $S f(M / S)$ as follows (see Appendix B):

$$
\begin{aligned}
V(S, M, \tau)=S\left(\frac{M}{S}\right)^{\tilde{\alpha}} e^{\tilde{\beta} \tau} \int_{1}^{\infty}[ & \psi\left(\ln \frac{M}{S}+\ln \xi, \tau\right)+\psi\left(\ln \frac{M}{S}-\ln \xi, \tau\right) \\
& \left.+2 \alpha \int_{\xi}^{\infty} \psi\left(\ln \frac{M}{S}+\ln \eta, \tau\right)\left(\frac{\eta}{\xi}\right)^{\tilde{\alpha}-1} d \eta\right] \frac{f(\xi)}{\xi \widetilde{\alpha}+2} d \xi
\end{aligned}
$$

where $\tilde{\beta}=-\left(1 / 2 \sigma^{2}\right)\left(r+\sigma^{2} / 2\right)^{2}$ and $\psi(x, \tau)$ are defined in $(2.4 \mathrm{~b})$. For the floating strike lookback option, we have $f(\xi)=\xi-1$. The corresponding price function is found to be (assuming $r>0$ )

$$
\begin{aligned}
V_{\mathrm{fl}}(S, M, \tau)= & M e^{-r \tau}\left[N(-d+\sigma \sqrt{\tau})-\frac{\sigma^{2}}{2 r}\left(\frac{M}{S}\right)^{2 r / \sigma^{2}} N\left(d-\frac{2 r}{\sigma} \sqrt{\tau}\right)\right] \\
& -S\left[N(-d)-\frac{\sigma^{2}}{2 r} N(d)\right],
\end{aligned}
$$

where $d=\left(\ln (S / M)+\left(r+\sigma^{2} / 2\right) \tau\right) / \sigma \sqrt{\tau}$.

Parity relation. The fixed strike lookback call option has payoff of the form $(M-K)^{+}$, where

$$
x^{+}= \begin{cases}x, & x>0 \\ 0, & \text { otherwise }\end{cases}
$$

while the payoff of the floating strike lookback put option takes the form $M-S$. Unlike the floating strike counterpart, the fixed strike payoff structure does not admit dimension reduction of the pricing model. Fortunately, there exists a parity relation between the price functions of fixed strike lookback call and floating strike lookback put (see Wong and Kwok's paper [5] for an alternative proof using the probability approach). 
Let $c_{\text {fix }}(S, M, \tau)$ and $p_{\mathrm{fl}}(S, M, \tau)$ denote the price function of the fixed strike lookback call option and the floating strike lookback put option, respectively. We define

$$
\tilde{V}(S, M, \tau)=c_{\mathrm{fix}}(S, M, \tau)-p_{\mathrm{fl}}(S, M, \tau)-S-K e^{-r \tau} .
$$

The governing equation for $\tilde{V}$ is given by

$$
\begin{aligned}
& \frac{\partial \tilde{V}}{\partial \tau}=\frac{\sigma^{2}}{2} S^{2} \frac{\partial^{2} \tilde{V}}{\partial S^{2}}+r S \frac{\partial \tilde{V}}{\partial S}-r \tilde{V}, \quad S>0, \tau>0, \\
& \left.\frac{\partial \tilde{V}}{\partial M}\right|_{S=M}=0, \quad \tau>0, \\
& \tilde{V}(S, M, 0)=(M-K)^{+}-(M-S)-(S-K) \\
& = \begin{cases}0 & \text { if } M \geq K, \\
K-M & \text { if } M<K .\end{cases}
\end{aligned}
$$

We claim that the solution to $\tilde{V}(S, M, \tau)$ is given by

$$
\tilde{V}(S, M, \tau)= \begin{cases}0 & \text { if } M \geq K, \\ p_{\mathrm{fl}}(S, K, \tau)-p_{\mathrm{fl}}(S, M, \tau) & \text { if } M<K .\end{cases}
$$

The solution observes continuity property at $M=K$; and the initial condition is satisfied since $p_{\mathrm{fl}}(S, K, 0)-p_{\mathrm{fl}}(S, M, 0)=(K-S)-(M-S)=K-M$. Also, $p_{\mathrm{fl}}(S, K, \tau)-$ $p_{\mathrm{fl}}(S, M, \tau)$ satisfies the governing equation together with the Neumann condition (note that $p_{\mathrm{fl}}(S, K, \tau)$ has no dependence on $\left.M\right)$. Hence, by uniqueness of a solution to problem (3.7), we obtain the following parity relation between $c_{\mathrm{fix}}$ and $p_{\mathrm{fl}}$ :

$$
\begin{aligned}
c_{\mathrm{fix}}(S, M, \tau) & = \begin{cases}p_{\mathrm{fl}}(S, M, \tau)+S-K e^{-r \tau} & \text { if } M \geq K, \\
p_{\mathrm{fl}}(S, K, \tau)+S-K e^{-r \tau} & \text { if } M<K\end{cases} \\
& =p_{\mathrm{fl}}(S, \max (M, K), \tau)+S-K e^{-r \tau} .
\end{aligned}
$$

\section{Conclusion}

The lookback option pricing models exhibit the interesting properties that the lookback variable does not appear explicitly in the governing equation, but only in the auxiliary conditions. The main contribution of this paper is the construction of an integral representation of the solution to pricing models with such degenerate feature. We demonstrate the use of the partial differential equation techniques to obtain integral price formulas for European lookback option models. We also deduce a parity relation between the price functions of floating strike and fixed strike lookback options.

\section{Appendices}

\section{A. Proof of (2.10)}

Observe that $W(x, \eta, u)$ is governed by (2.2). We multiply each term in the equation by $G(-\eta, \tau-u ; z-x)$, then integrate from $x=0$ to $x=\infty$ and from $u=0$ to $u=\tau-\epsilon$ 
( $\epsilon$ is a small positive constant) to obtain

$$
\begin{aligned}
0= & \int_{0}^{\tau-\epsilon} \int_{0}^{\infty} G(-\eta, \tau-u ; z-x) \frac{\partial W}{\partial u}(x, \eta, u) d x d u \\
& -\frac{\sigma^{2}}{2} \int_{0}^{\tau-\epsilon} \int_{0}^{\infty} G(-\eta, \tau-u ; z-x) \frac{\partial^{2} W}{\partial x^{2}}(x, \eta, u) d x d u \\
& +\left(r-\frac{\sigma^{2}}{2}\right) \int_{0}^{\tau-\epsilon} \int_{0}^{\infty} G(-\eta, \tau-u ; z-x) \frac{\partial W}{\partial x}(x, \eta, u) d x d u
\end{aligned}
$$

By performing part integration and applying the homogeneous boundary condition $W(0, \eta, u)=0$, we obtain

$$
\begin{aligned}
0= & \int_{0}^{\infty}[G(-\eta, \epsilon ; z-x) W(x, \eta, \tau-\epsilon)-G(-\eta, \tau ; z-x)] W(x, \eta, 0) d x \\
+ & \frac{\sigma^{2}}{2} \int_{0}^{\tau-\epsilon} G(-\eta, \tau-u ; z-x) \frac{\partial W}{\partial x}(0, \eta, u) d u \\
+ & \int_{0}^{\tau-\epsilon} \int_{0}^{\infty}\left[\frac{\partial G}{\partial \tau}(-\eta, \tau-u ; z-x)-\frac{\sigma^{2}}{2} \frac{\partial^{2} G}{\partial z^{2}}(-\eta, \tau-u ; z-x)\right. \\
& \left.+\left(r-\frac{\sigma^{2}}{2}\right) \frac{\partial G}{\partial z}(-\eta, \tau-u ; z-x)\right] W(x, \eta, u) d x d u .
\end{aligned}
$$

Next, we take the limit $\epsilon \rightarrow 0^{+}$and observe that

$$
\begin{gathered}
\lim _{\epsilon \rightarrow 0^{+}} G(-\eta, \epsilon ; z-x)=\delta(x-z-\eta), \\
G(-\eta, \tau ; z-x)=G(x, \tau ; z+\eta),
\end{gathered}
$$

we then obtain

$$
\begin{aligned}
\int_{0}^{\tau} G( & -\eta, \tau-u ; z) \frac{\sigma^{2}}{2} \frac{\partial W}{\partial x}(0, \eta, u) d u \\
& =\int_{0}^{\infty} G(-\eta, \tau ; z-\xi) W(\xi, \eta, 0) d \xi-H(z+\eta) W(z+\eta, \eta, \tau) \\
& =\int_{0}^{\infty}\{[1-H(z+\eta)] G(\xi, \tau ; z+\eta)+H(z+\eta) \hat{G}(\xi, \tau ; z+\eta)\} W(\xi, \eta, 0) d \xi
\end{aligned}
$$

where $H(x)$ is the Heaviside function. Next, we integrate each term in the above equation with respect to $\eta$ over the interval $(-\infty, \infty)$ to give

$$
\begin{aligned}
\int_{0}^{\tau} \int_{-\infty}^{\infty} G(-\eta, \tau-u ; z) \frac{\sigma^{2}}{2} \frac{\partial W}{\partial x}(0, \eta, u) d \eta d u \\
=\int_{0}^{\infty} \int_{-\infty}^{-z} G(\xi, \tau ; z+\eta) W(\xi, \eta, 0) d \eta d \xi \\
\quad+\int_{0}^{\infty} \int_{-z}^{\infty} \hat{G}(\xi, \tau ; z+\eta) W(\xi, \eta, 0) d \eta d \xi
\end{aligned}
$$

where

$$
\widehat{G}(\xi, \tau ; x)=e^{\alpha(x-\xi)+\beta \tau} \psi(\xi+x, \tau) .
$$


124 Integral price formulas for lookback options

Substituting the above relations into (2.6), we have

$$
\begin{aligned}
V(x, y, \tau)= & \int_{0}^{\infty} \int_{-\infty}^{y} G(\xi, \tau ; x+\eta-y) W(\xi, \eta, 0) d \eta d \xi \\
& +\int_{0}^{\infty} \int_{y}^{\infty} \hat{G}(\xi, \tau ; x+\eta-y) W(\xi, \eta, 0) d \eta d \xi \\
& +\int_{-\infty}^{\infty} G(0, \tau ; x+\eta-y) W(0, \eta, 0) d \eta
\end{aligned}
$$

Lastly, by applying the following relations and performing part integration:

$$
\begin{gathered}
W(\xi, \eta, 0)=\left(\frac{\partial}{\partial \xi}+\frac{\partial}{\partial \eta}\right) V_{T}\left(e^{\eta-\xi}, e^{\eta}\right) \\
\frac{\partial G}{\partial \xi}(\xi, \tau ; x+\eta-y)=-\frac{\partial G}{\partial \eta}(\xi, \tau ; x+\eta-y), \\
\left(\frac{\partial}{\partial \xi}+\frac{\partial}{\partial \eta}\right) \hat{G}(\xi, \tau ; x+\eta-y)=\left(\frac{\partial}{\partial \xi}+\frac{\partial}{\partial \eta}\right) \bar{G}(\xi, \tau ; x+\eta-y),
\end{gathered}
$$

we obtain

$$
\begin{aligned}
V(x, y, \tau)= & \int_{0}^{\infty} \bar{G}(\xi, \tau ; x) V_{T}\left(e^{y-\xi}, e^{y}\right) d \xi \\
& +\int_{0}^{\infty} \int_{y}^{\infty}\left[\left(\frac{\partial}{\partial \xi}+\frac{\partial}{\partial \eta}\right) \bar{G}(\xi, \tau ; x+\eta-y)\right] V_{T}\left(e^{\eta-\xi}, e^{\eta}\right) d \eta d \xi
\end{aligned}
$$

Transforming back to the original variables $S$ and $M$, we obtain the result in (2.10).

\section{B. Proof of (3.3)}

Suppose we set $U(x, \tau)=\tilde{U}(x, \tau) e^{\tilde{\alpha} x+\tilde{\beta} \tau}$, where $\tilde{\alpha}=r / \sigma^{2}+1 / 2$ and $\tilde{\beta}=-\left(1 / 2 \sigma^{2}\right)\left(r+\sigma^{2} / 2\right)^{2}$, then $\tilde{U}(x, \tau)$ is governed by

$$
\begin{gathered}
\frac{\partial \tilde{U}}{\partial \tau}=\frac{\sigma^{2}}{2} \frac{\partial^{2} \tilde{U}}{\partial x^{2}}, \quad x>0, \tau>0, \\
\left.\left(\frac{\partial \tilde{U}}{\partial x}+\tilde{\alpha} \tilde{U}\right)\right|_{x=0}=0, \quad \tau>0, \\
\tilde{U}(x, 0)=e^{-\tilde{\alpha} x} f\left(e^{x}\right)=h_{+}(x), \quad x>0 .
\end{gathered}
$$

Let $h_{-}(x)$ denote the continuation of the initial condition for $x<0, \tilde{U}(x, \tau)$ can then be formally represented by

$$
\tilde{U}(x, \tau)=\int_{-\infty}^{0} \psi(x-\xi, \tau) h_{-}(\xi) d \xi+\int_{0}^{\infty} \psi(x-\xi, \tau) h_{+}(\xi) d \xi,
$$

where $\psi(x, \tau)$ is defined in $(2.4 \mathrm{~b})$. The function $h_{-}(x)$ is determined by enforcing the satisfaction of the Robin boundary condition (B.2a) by the solution $\tilde{U}(x, \tau)$ in (B.3). We 
then obtain the following governing differential equation for $h_{-}(x)$ :

$$
\begin{gathered}
h_{-}^{\prime}(x)+\tilde{\alpha} h_{-}(x)+h_{+}^{\prime}(-x)+\tilde{\alpha} h_{+}(-x)=0, \\
h_{+}(0)=h_{-}(0) .
\end{gathered}
$$

For example, suppose $f\left(e^{x}\right)=e^{x}-1$, then $h_{+}(x)=e^{-\widetilde{\alpha} x}\left(e^{x}-1\right)$. By solving (B.4), we obtain

$$
h_{-}(x)=\frac{e^{-\tilde{\alpha} x}-e^{(\widetilde{\alpha}-1) x}}{2 \widetilde{\alpha}-1} .
$$

In general, the solution to (B.4) is found to be

$$
h_{-}(x)=h_{+}(-x)+2 \tilde{\alpha} e^{-\tilde{\alpha} x} \int_{0}^{x} e^{\tilde{\alpha} \xi} h(-\xi) d \xi
$$

Substituting the above expression for $h_{-}(x)$ into (B.3) and performing some simplification, we obtain (3.3).

\section{Acknowledgment}

The authors thank Professor Lishang Jiang for his constructive comments during the course of the research.

\section{References}

[1] G. Barles, Convergence of numerical schemes for degenerate parabolic equations arising in finance theory, Numerical Methods in Finance (L. C. G. Rogers and D. Talay, eds.), Publ. Newton Inst., Cambridge University Press, Cambridge, 1997, pp. 1-21.

[2] A. Conze and Viswanathan, Path dependent options: the case of lookback options, J. Finance 46 (1991), 1893-1907.

[3] M. B. Goldman, H. B. Sosin, and M. A. Gatto, Path dependent options: buy at the low, sell at the high, J. Finance 34 (1979), 1111-1127.

[4] H. He, W. P. Keirstead, and J. Rebholz, Double lookbacks, Math. Finance 8 (1998), no. 3, 201228.

[5] H. Y. Wong and Y. K. Kwok, Sub-replication and replenishing premium: efficient pricing of multistate lookbacks, Rev. Deriv. Res. 6 (2003), 83-106.

Chenglong Xu: Department of Applied Mathematics, Tongji University, Shanghai 200092, China

E-mail address: clxu601@online.sh.cn

Yue Kuen Kwok: Department of Mathematics, The Hong Kong University of Science and Technology, Kowloon, Hong Kong

E-mail address: maykwok@ust.hk 


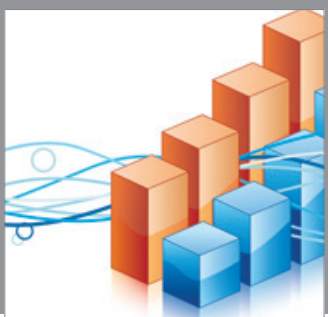

Advances in

Operations Research

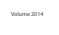

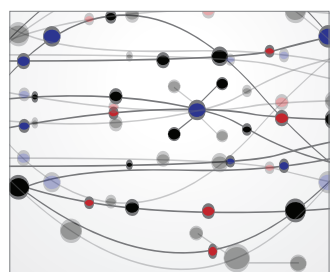

\section{The Scientific} World Journal
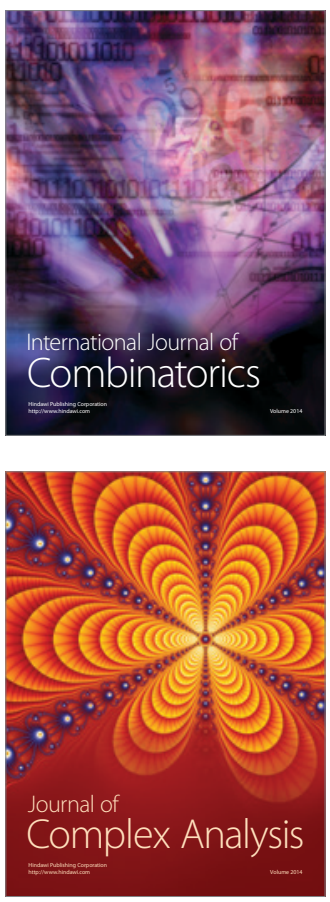

International Journal of

Mathematics and

Mathematical

Sciences
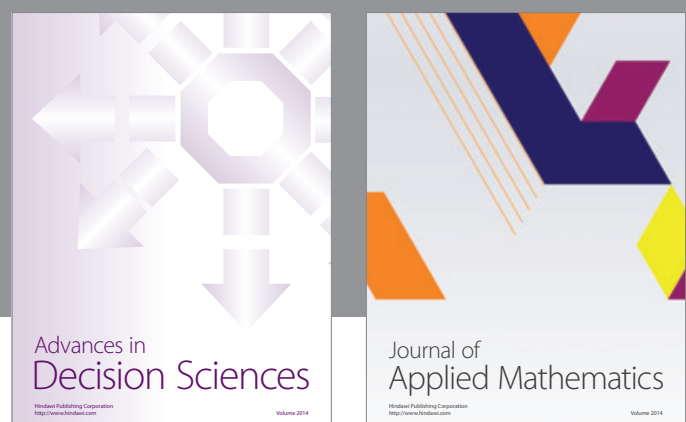

Journal of

Applied Mathematics
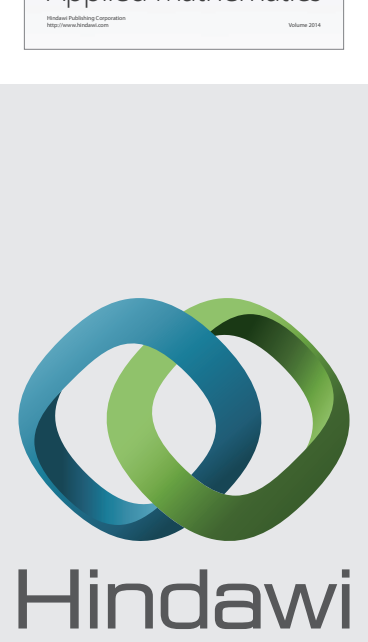

Submit your manuscripts at http://www.hindawi.com
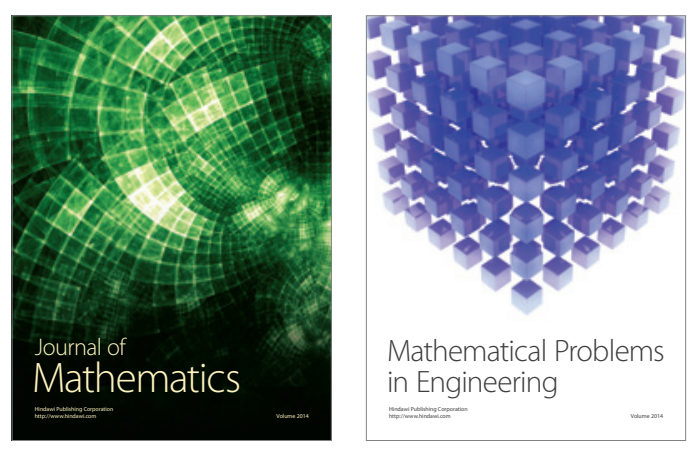

Mathematical Problems in Engineering
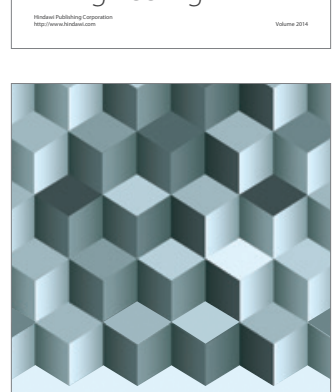

Journal of

Function Spaces
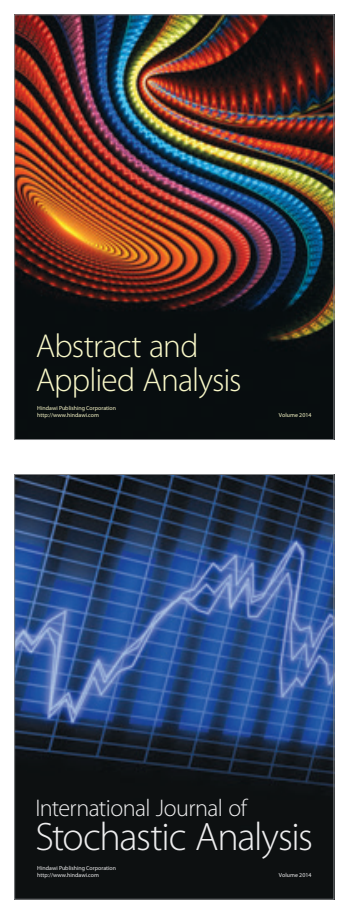

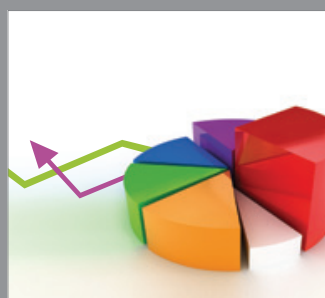

ournal of

Probability and Statistics

Promensencen
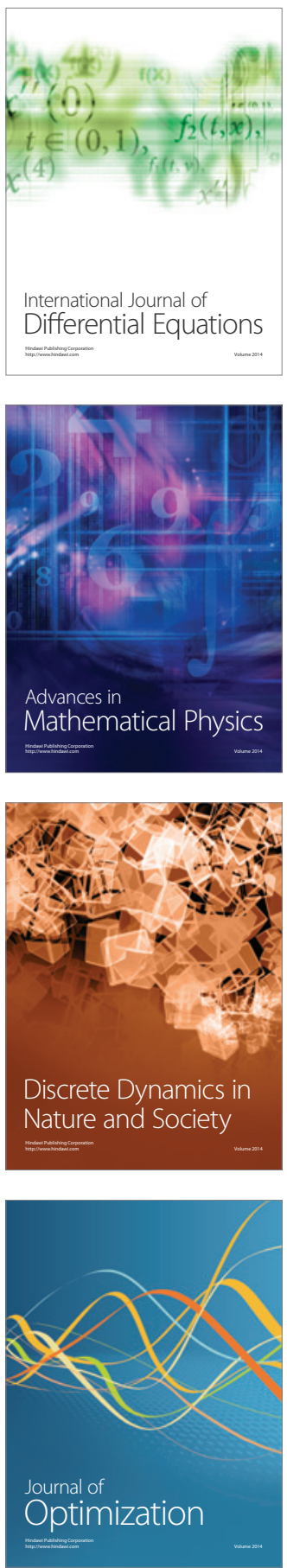